\title{
Prediction of No-Reflow Phenomenon After Successful Percutaneous Coronary Intervention in Patients With Acute Myocardial Infarction
} Intravascular Ultrasound Findings

\author{
Tetsuya Watanabe, MD*; Shinsuke Nanto, MD; Masaaki Uematsu, MD; Tomoki Ohara, MD; \\ Takakazu Morozumi, MD; Jun-ichi Kotani, MD; Mayu Nishio, MD; Masaki Awata, MD; \\ Seiki Nagata, MD; Masatsugu Hori, MD*
}

\begin{abstract}
Plaque characterization by intravascular ultrasound (IVUS) before percutaneous coronary intervention (PCI) was evaluated in 81 consecutive patients with acute myocardial infarction (AMI) to establish if IVUS can predict the occurrence of the 'no-reflow' phenomenon. Angiographic no-reflow was defined as TIMI flow grade 1 or 2 without any mechanical obstruction in the epicardial artery. Patients were divided into 2 groups according to the post-PCI angiograms: normal flow (group $\mathrm{R}, \mathrm{n}=60$ ) and no-reflow (group NR, $\mathrm{n}=21$ ). Although the incidence of either soft or noncalcified plaque was not statistically different between the groups, positive vessel remodeling was more frequent in group NR than in group $\mathrm{R}(57.1 \% \mathrm{vs} 31.6 \%, \mathrm{p}<0.05)$. Lipid core was also more frequently found in group NR than in group R $(61.9 \%$ vs $25.0 \%$, p $<0.01)$. Positively remodeled vessels with lipid-rich plaques as characterized by IVUS before PCI predicted the occurrence of angiographic no reflow with a sensitivity of 43\% and a specificity of 60\%. (Circ J 2003; 67: 667-671)
\end{abstract}

Key Words: Acute myocardial infarction; Intravascular ultrasound; Lipid core; No-reflow; Remodeling

$\mathbf{P}$ ercutaneous coronary intervention (PCI), if performed adequately, limits infarct size and improve prognosis in patients with acute myocardial infarction (AMI)!, Patency of the infarct-related artery, however, does not always guarantee restoration of normal coronary blood flow. This 'no-reflow' phenomenon is related to a poor clinical outcome, and although a considerable number of patients with AMI who undergo successful PCI are at risk of no-reflow, the mechanisms remain unclear and reliable prediction of the occurrence of no-reflow before PCI is performed has not been established!

No-reflow is frequently encountered in the setting of elective PCI for degenerated, plaque-rich saphenous vein graft stenoses, ${ }^{5,6}$ Hence, we postulated that the plaque characteristics of the infarct-related coronary artery lesion may be closely related to the occurrence of no-reflow?-12 However, intracoronary plaque characterization is not possible using coronary angiography alone. Recently, intravascular ultrasound (IVUS) has emerged as a powerful tool for the evaluation of coronary artery lesion morphology, ${ }^{13}$ so in the present study we decided to perform IVUS prior to PCI in patients with AMI to characterize the infarct-related coronary artery lesion morphology and investigate its relationship to the angiographic no-reflow phenomenon.

(Received February 13, 2003; revised manuscript received April 16, 2003; accepted May 12, 2003)

Cardiovascular Division, Kansai Rosai Hospital and *Department of Medical Therapeutics, Osaka University Graduate School of Medicine, Osaka, Japan

Mailing address: Tetsuya Watanabe, MD, Department of Medical Therapeutics, Osaka University Graduate School of Medicine, 2-2 Yamadaoka, Suita, Osaka 565-0871, Japan

\section{Methods}

\section{Study Population}

The study prospectively enrolled 81 consecutive patients with AMI (58 men, 23 women) who underwent emergency PCI from May 2000 through October 2001. The Ethics Committee of Kansai Rosai Hospital approved the study protocol and all patients gave written informed consent. Clinical data including age, gender, risk factors for coronary artery disease (eg, diabetes mellitus, hypertension, total cholesterol, triglyceride, blood pressure, smoking history, previous MI) were collected. Patients satisfying all of the following criteria were given a diagnosis of AMI: (1) chest pain $\geq 30$ min duration; (2) ST elevation $\geq 0.1 \mathrm{mV}$ in at least 2 adjacent leads on ECG; (3) increase in creatine kinase (CK) concentration to double the normal value. The examined vessels were 44 left anterior descending arteries, 28 right coronary arteries and 9 left circumflex arteries.

\section{Angiographic Definition of No-Reflow}

Coronary flow was angiographically assessed by TIMI flow grade, as defined in the Thrombolysis in Myocardial Infarction (TIMI) trial ${ }^{14}$ Flow reduction includes both slow flow and no-reflow. No-reflow was defined in this study as transient or permanent flow reduction as compared with the flow obtained immediately after aspiration with a Rescue ${ }^{\mathrm{TM}}$ catheter, equal to or more than TIMI grade 1 that was neither attributable to abrupt closure, severe dissection of the original target lesion, nor epicardial spasm. Slow flow or TIMI grade 2 even after successful PCI was also included as no-reflow. The average TIMI flow grade among all patients was 0.56 initially, 0.97 after wire crossing, 2.26 after 


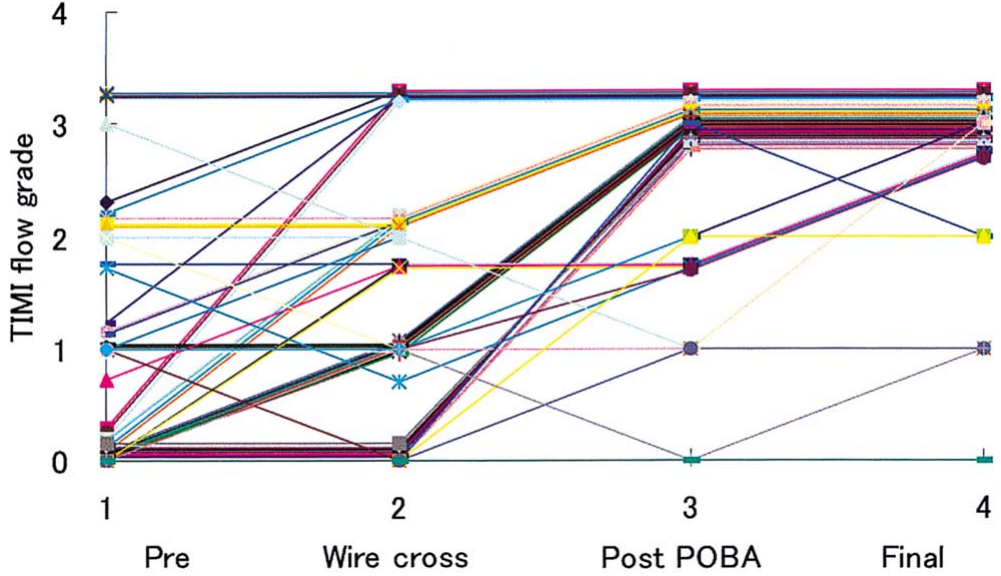

Fig 1. TIMI flow during PCI procedures. TIMI, Thrombolysis in Myocardial Infarction; Pre, initial angiogram; Wire cross, immediately after the fist wire crossing; POBA, plain old balloon angioplasty.

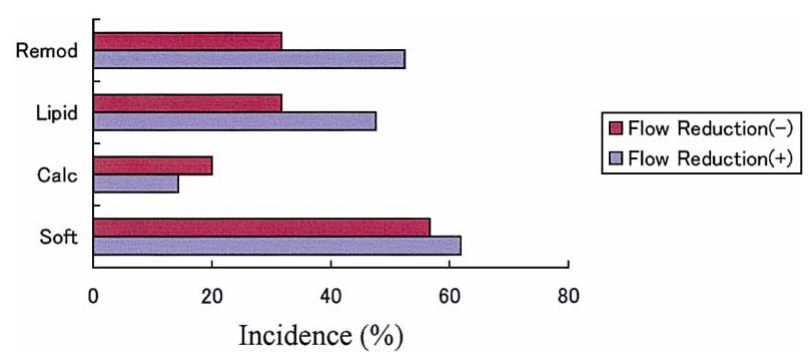

Fig 2. Comparison of the plaque characterization by IVUS in patients with and without the no-reflow phenomenon. Remod, vessel remodeling; Lipid, lipid core; Calc, superficial calcification; Soft, soft plaque.

Rescue, 2.38 after PCI, and was 2.69 by final angiography (Fig 1). Although the overall TIMI flow grade increased after the PCI procedure, TIMI flow grade worsened in some patients after PCI despite additional balloon dilatations. Angiographic no-reflow was found in 21 cases (flow reduction: 11 cases; final no-reflow: 10 cases). Patients were accordingly divided into 2 groups based on the postPCI angiograms: angiograms with normal flow (group R, $\mathrm{n}=60$ ) and those with no-reflow (group NR, $n=21$ ).

\section{IVUS and PCI Procedures}

For diagnostic coronary angiography, intravenous heparin (3,000 units) and intracoronary isosorbide dinitrate $(0.4-0.6 \mathrm{mg})$ were administered, and the right and left coronary arteries were imaged by the femoral artery approach using a $5 \mathrm{Fr}$ catheter to identify the infarct-related coronary artery. After the routine diagnostic catheterization was completed, an 8Fr guide catheter was inserted into the responsible coronary artery followed by an additional 7,000 units of heparin. Next, a 0.014-inch guidewire was passed through the lesion. According to the hospital's strategy for the treatment of AMI, a Rescue ${ }^{\mathrm{TM}}$ catheter was used to aspirate as many intracoronary thrombi as possible to prevent distal embolization. On completion of the aspiration, in all patients underwent IVUS before the PCI procedure. An ultrasound transducer $(30 \mathrm{MHz}, 3.2 \mathrm{~F}$, Ultracross, Boston Scientific Corporation, Natick, MA, USA) was advanced as distally as possible over the guidewire and withdrawn by both slow manual and auto pullback $(1 \mathrm{~mm} / \mathrm{s})$ methods. While pulling back the catheter, we manually infused a contrast medium to exclude the residual thrombus by improving the lumen/vessel contrast. Ultrasound images were
Table 1 Patient Characteristics

\begin{tabular}{lccc}
\hline \hline & No-reflow (-) & No-reflow $(+)$ & $p$ value \\
\hline Age (years) & 64.7 & 65.2 & $N S$ \\
M/F (\%) & $42 / 18(30 \%)$ & $16 / 5(23.8 \%)$ & $N S$ \\
Previous MI & $5(8.3 \%)$ & $3(14.2 \%)$ & $N S$ \\
Previous CABG & $5(8.3 \%)$ & $3(14.2 \%)$ & $N S$ \\
Cardiogenic shock & $3(5.0 \%)$ & $3(14.2 \%)$ & $N S$ \\
Peak CK & $3378 \pm 2761$ & $3776 \pm 3107$ & $N S$ \\
Peak CK-MB & $293 \pm 222$ & $336 \pm 266$ & $N S$ \\
Diabetes mellitus & $15 / 60(25 \%)$ & $6 / 21(28.5 \%)$ & $N S$ \\
Hypertension & $25 / 60(41.7 \%)$ & $13 / 21(61.9 \%)$ & $N S$ \\
Hyperlipidemia & $20 / 60(33.3 \%)$ & $9 / 21(42.9 \%)$ & $N S$ \\
Smoking & $34 / 60(56.7 \%)$ & $12 / 21(57.1 \%)$ & $N S$ \\
Final stent use & $17 / 60(28.3 \%)$ & $13 / 21(61.9 \%)$ & $<0.01$ \\
\hline
\end{tabular}

$C A B G$, coronary artery bypass grafting; $C K$, creatine kinase; $M I$, myocardial infarction.

recorded on videotapes.

Cineangiographic documentation of the lesion location was used for site identification! 15

\section{IVUS Image Analysis}

After the procedure, the ultrasound images were analyzed using computer software (3D Netra IVUS, Scimage Inc, CA, USA). The culprit lesion site and a proximal reference site were selected for measurement. The culprit lesion was defined as the site with the smallest lumen diameter on the IVUS images. Lumen area was determined by tracing the boundary between the lumen and the leading edge of the intima, excluding residual thrombus. Total vessel area (TVA) was determined by tracing the leading edge of the media-adventitia boundary. Plaque area was calculated as: total vessel area-lumen area. Percent plaque area was computed using the formula: \{ (total vessel area-lumen area)/total vessel area $\times 100$. Plaque morphology was classified into 3 types: soft, hard and calcified. A soft plaque was defined as plaque that was less echogenic than the reference adventitia; hard plaque produced a similar level of echo intensity as the adventitia; and calcified lesions were defined as echogenic plaque with acoustic shadowing occupying $>90^{\circ}$ of the vessel wall circumference.16,17 The remodeling index (RI) was defined as the ratio of the TVA at the lesion site to the TVA at the proximal reference site: $\mathrm{RI}>1.05$ was regarded as positive remodeling. 
(c)

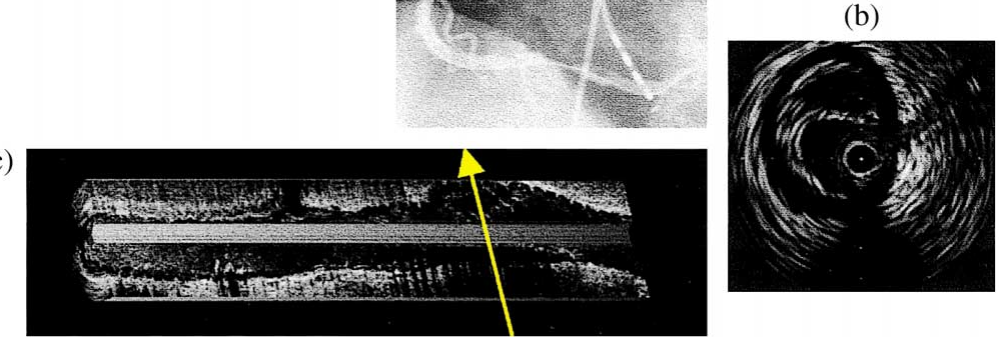

(a)

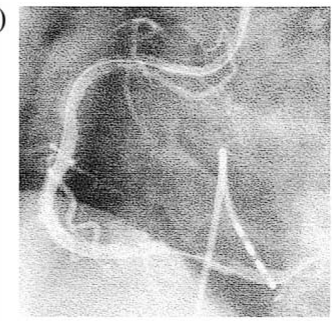

Fig 3. Representative coronary angiography and IVUS images immediately after thrombectomy in a patient with the noreflow phenomenon. (a) Right coronary angiogram showing lumen irregularities after thrombectomy by Rescue ${ }^{\mathrm{TM}}$ catheter. (b) Original IVUS image from the site of the arrow in (c). A large, remodeled vessel containing a large lipid core can be seen. (c) Reconstructed 3-D IVUS image of the culprit lesion (distal segment of the right coronary artery) showing a large remodeled vessel with a lipid core.

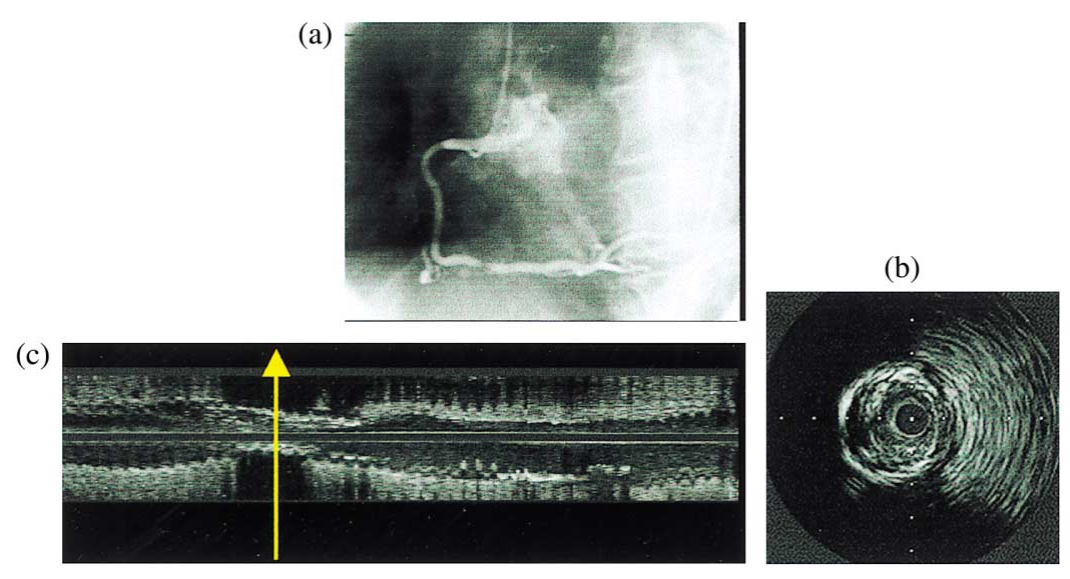

Fig 4. Representative IVUS images immediately after thrombectomy in a patient with good reflow (TIMI 3). (a) Right coronary angiogram after thrombectomy by Rescue ${ }^{\mathrm{TM}}$ catheter. (b) Original IVUS image from the distal segment of the right coronary artery. (c) Reconstructed 3-D IVUS image showing calcification without vessel remodeling.

\section{Statistical Analysis}

Numerical data were expressed as mean $\pm \mathrm{SD}$. Chi-square analysis was used to assess the difference among categorical variables. Student's t-test was used to assess differences in numerical data. In this stud $\mathrm{p}<0.05$ was considered to be significant.

\section{Results}

\section{Patient Characteristics}

Background characteristics including age, gender, responsible vessel, and coronary risk factors (eg, diabetes mellitus, hypertension, hyperlipidemia, and smoking) were not different between group $\mathrm{R}$ and group NR, except for the stent use. Lesions could be crossed with the Rescue ${ }^{\mathrm{TM}}$ catheter in 78 patients $(96.3 \%$ ), except for 3 patients, thereby eliminating the residual thrombus at the culprit lesion in the majority of patients. Stents were more frequently used in group NR than in group R $(61.9 \%$ vs $28.3 \%, \mathrm{p}<0.01$, Table 1). Multiple complex coronary stenoses were found $18(22.2 \%)$ of the culprit lesions.

\section{Quantitative IVUS Measurements}

A calcified lesion was found in 15 cases (18\%), soft plaque in 47 cases $(58 \%)$ and lipid core in 29 cases $(35 \%)$. Positive vessel remodeling, as represented in Fig 3, was found in 30 cases (37\%). Positive vessel remodeling was more frequent in group NR than in group $\mathrm{R}(57.1 \%$ vs $31.6 \%, \mathrm{p}<0.05)$. A lipid core was also more frequently found in group NR than in group $\mathrm{R}(61.9 \%$ vs $25.0 \%$, $\mathrm{p}<0.01)$. Calcification tended to be less frequent in group NR, although it did not reach statistical significance. The presence of both vessel remodeling and a lipid core on IVUS predicted angiographic no-reflow with a sensitivity of $43 \%$ and specificity of $60 \%$.

\section{Discussion}

We demonstrated in this study that the occurrence of angiographic no-reflow can be predicted by IVUS performed before emergency PCI in patients with AMI. The presence of both positive vessel remodeling and a lipid core was the major predictor of angiographic no-reflow! 18 The no-reflow phenomenon has been attributed to multiple factors including damage to the endothelial cells, tissue 
edema ${ }^{19}$ neutrophil plugging of the microvessels ${ }^{20,21}$ microvascular spasm ${ }_{2}^{22,23}$ oxygen-free radicals ${ }^{24,25}$ embolization caused by the thrombus burden at culprit lesion, and distal embolization by plaque from the culprit lesion. Considering the experience reported from elective PCI for degenerated saphenous vein grafts, which are often associated with large plaques, 4,26 distal embolization by materials from the lesion site may play an important role in the occurrence of no-reflow in the acute setting. Thus, it can be anticipated that there is a close relationship between lesion morphology and the incidence of angiographic no-reflow. Accordingly, we used IVUS to characterize lesion morphology before the PCI procedure. Although the lesion vessel area was similar between group NR and group $\mathrm{R}$ $\left(15.3 \pm 5.7 \mathrm{~mm}^{2}\right.$ vs $14.4 \pm 4.7 \mathrm{~mm}^{2}$, NS), vessel remodeling was more significant in group NR than in group R $(57.1 \%$ vs $31.6 \%, \mathrm{p}<0.05)$. The present study thus demonstrated that both lesions that are lipid rich and remodeled large plaques are associated with the development of angiographic no-reflow.

We performed thrombus aspiration prior to IVUS to exclude as much as possible the occurrence of distal embolization by intracoronary thrombi. Nevertheless, noreflow still occurred in 21 cases $(25.9 \%)$, which strongly suggests that the plaque contents, which will be destroyed during the PCI procedure, are also important in the occurrence of no-reflow, in addition to thrombus.

The characterization of plaque was done by IVUS in this study, but although this methodology is well established, it cannot always distinguish thrombi from underlying plaque, and its accuracy depends on the instrument settings and the expertise of the interpreter. Because of this limitation, we used a Rescue ${ }^{\mathrm{TM}}$ thrombectomy catheter before IVUS and PCI to eliminate thrombi as much as possible? 7

Tanaka et al have independently reported that large vessels associated with a lipid pool-like IVUS image indicate a high risk for no-reflow after PCI for AMI, also suggesting that plaque content may play a role in the occurrence of noreflow 28 In their study, however, distal embolization by the thrombus burden at the lesion site was not sufficiently ruled out.

\section{Study Limitations}

The no-reflow phenomenon was angiographically defined in this study; however, the microcirculation was not directly evaluated, for example, by contrast echocardiography. Hence, the mechanism of the phenomenon remains speculative. Classification of plaque morphology is based on a computer-assisted visual interpretation, which depends on the instrument settings and the expertise of the interpreter. Although aspiration with a Rescue ${ }^{\mathrm{TM}}$ catheter had been extensively performed prior to IVUS and PCI, intracoronary thrombi may not have been completely eliminated.

\section{Clinical Implications}

New PCI strategies (eg, distal protection devices) could be used to prevent the no-reflow phenomenon if its occurrence can be predicted prior to PCI in patients with AMI.

In conclusion, the presence of both lipid core and vessel remodeling without calcification on IVUS are possible predictors of the occurrence of angiographic no-reflow after PCI in patients with AMI. Destruction of the plaque and subsequent distal embolization may be a cause of noreflow.

\section{References}

1. Simes RJ, Topel EJ, Holmer DR Jr, White HD, Rutsch WR, Vahanian A, et al for the GUSTO-1 Investigators. Link between the angiographic substudy and mortality outcomes in a large randermized trial of myocardial reperfusion: Importance of early and complete infarct artery reperfusion. Circulation 1995; 91: 1923-1928.

2. Nagao K, Hayashi N, Kanmatsuse K, Kikuchi S, Ohuba T, Takahashi H. An early and complete reperfusion strategy for acute myocardial infarction using fibrinolysis and subsequent transluminal therapy: The FAST trial. Circ J 2002; 66: 576-582.

3. Anderson JL, Karagounis L, Becker LC, Sorensen SG, Menlove RL, for the TEAM-3 Investigators. TIMI perfusion grade 3 but not grade 2 results in improved outcome after thrombolysis for myocardial infarction: Ventriculographic, enzymatic, and electrocardiographic evidence from the TEAM-3 study. Circulation 1993; 87: 18291839.

4. Piana RN, Paik GY, Moscucci M, Cohen DJ, Gibson CM, Kugelmass $\mathrm{AD}$, et al. Incidence and treatment of no reflow after percutaneous coronary intervention. Circulation 1994; 89: 2514-2518.

5. Webb JG, Carere RG, Virmani R, Baim D, Teirstein PS, Whitlow P, et al. Retrieval and analysis of particulate debris after saphenous vein graft intervention. J Am Coll Cardiol 1999; 34: 468-475.

6. Watson PS, Hadjipetrou P, Cox SV, Pyne CT, Gossman DE, Piemonte TC, et al. Angiografhic and clinical outcomes following acute infarct angioplasty on saphenous vein grafts. Am J Cardiol 1999; 83: 1018-1021.

7. White HD, Norris RM, Brown MA, Takayama M, Maslowski A, Bass NM, et al. Effect of intravenous streptokinase on left ventricular function and early survival after acute myocardial infarction. $N$ Engl J Med 1987; 317: 850-855.

8. Ito H, Tomooka T, Sakai N, Yu H, Higashino Y, Fujii K, et al. Lack of myocardial perfusion immediately after successful thrombolysis: A predictor of poor recovery of left ventricular function in anterior myocardial infarction. Circulation 1992; 85: 1699-1705.

9. Grines CL, Browne KF, Macro J, Ruthbaum D, Stone GW, O'Keefe $\mathrm{J}$, et al. A comparison of immediate angioplasty with thrombolytic therapy for acute myocardial infarction. N Engl J Med 1993; 328: $673-679$.

10. The GUSTO angiographic investigators. The effects of tissue plasminogen activator, streptokinase, or both on coronary-artery patency, ventricular function, and survival after acute myocardial infarction. N Engl J Med 1993; 329: 1615-1622.

11. Morishima I, Sone T, Mokuno S, Taga S, Shimauchi A, Oki Y, et al. Clinical significance of no-reflow phenomenon observed on angiography after successful treatment of acute myocardial infarction with percutaneous transluminal coronary angioplasty. Am Heart J 1995; 130: $239-243$.

12. Ames A, Wright RL, Kowada M, Thurston JM, Majno G. The noreflow phenomenon. Am J Pathol 1968; 52: 437-447.

13. Yamagishi M, Hosokawa H, Saito S, Kanemitsu S, Chino M, Koyanagi S, et al. Coronary disease morphorogy and distribution determined by quantitative angiography and intravascular ultrasound: Re-evaluation in a Cooperative Multicenter Intravascular Ultrasound Study (COMIUS). Circ J 2002; 66: 753-740.

14. Cheesebro JH, Knatterud G, Roberts R, Borer J, Cohen LS, Dalen J, et al. Thrombolysis in myocardial infarction (TIMI) trial. Phase Ö̈̈: A comparison between intravenous plasminogen activator and intravenous streptokinase: Clinical findings through hospital discharge. Circulation 1987; 76: $142-154$.

15. Nishimura RA, Edwards WD, Warnes CA, Reeder GS, Holmes DR Jr, Tajik AJ, et al. Intravascular ultrasound imaging: In vitro validation and pathologic correlation. J Am Coll Cardiol 1990; 16: 145154.

16. Goldstein JA, Demetriou D, Grines CL, Pica M, Shoukfeh M, O'Neill WW. Multiple complex coronary plaques in patients with acute myocardial infarction. N Engl J Med 2000; 28: 915-922.

17. Tobis JM, Mallery J, Mahon D, Lehmann K, Zalesky P, Griffith J, et al. Intravascular ultrasound imaging of human coronary arteries in vivo: Analysis of tissue characterizations with comparison to invitro histological specimens. Circulation 1991; 83: 913-926.

18. Kloner RA, Ganote CE, Jennings RB. The no-reflow phenomenon after temporary coronary occlusion in the dog. J Clin Invest 1974; 54: $1496-1508$.

19. Kotani J, Nanto S, Kitakaze M, Ohara T, Morozumi T, Watanabe T, Fujita M, Hori M, Nagata S. No-reflow followingdilatation of a coronary lesion with a large lipid core. Circ J 2002; 66: 702-704.

20. Engler RL, Schmid-schonbein GW, Pavelec RS. Leukocyte capillary plugging in myocardial ischemia and reperfusion in the dog. Am J Pathol 1983; 111: 98-111. 
21. Meisel SR, Shapiro H, Radney J, Neuman Y, Khaskia AR, Gruener $\mathrm{N}$, et al. Increased expression of neutrophil and monocyte adhesion molecules LFA-1 and Mac-1 and their ligand ICAM- 1 and VLA-4 throughout the acute phase of myocardial infarction: Possible implications for leukocyte aggregation and microvascular plugging. $J \mathrm{Am}$ Coll Cardiol 1998; 31: 120-125.

22. Piana RN, Paik GY, Moscucci M, Cohen DJ, Gibson CM, Kugelmass AD, et al. Incidence and treatment of 'no-reflow' after percutaneous coronary intervention. Circulation 1994; 89: 2514 2518.

23. Taniyama Y, Ito H, Iwakura K, Masuyama T, Hori M, Takiuchi S, et al. Beneficial effect of intracoronary verapamil on microvascular and myocardial salvage in patients with acute myocardial infarction. $J$ Am Coll Cardiol 1997; 30: 1193-1199.

24. Kloner RA, Przylenk K, Wittaker P. Deleterious effect of oxygen radicals in ischemic/reperfusion: Resolved and unsolved issues. Circulation. 1989; 80: 1115-1127.
25. Grech ED, Dodd NJF, Jackson MJ, Morrison WL, Faragher EB, Ramsdale DR, et al. Evidence for free radical generation after primary percutaneous transluminal coronary angioplasty recanalization in acute myocardial infarction. Am J Cardiol. 1996; 77: $122-$ 127.

26. Abbo KM, Dooris M, Glazier S. Features and outcome of no-reflow after percutaneous coronary intervention. Am J Cardiol. 1995; 75: $778-782$.

27. Watanabe T, Nanto S, Ohara T, Nishio M, Awata M, Yoshida M, et al. Predictors of angiographic 'no-reflow' phenomenon in patients with acute myocardial infarction: A serial intravascular ultrasound study (abstract). Circulation 2001; (Suppl) 3662.

28. Tanaka, A, Kawarabayashi T, Nishibori Y, Sano T, Nishida Y, Fukuda D, et al. No-reflow phenomenon and lesion morphology in patients with acute myocardial infarction. Circulation. 2002; 105: $2148-2152$. 